\title{
Index
}

Note: Literary works by Marx and Engels can be found under their names.

abolitionism 20-22, 24-25, 28, $32-33,36-38,77,250$

see also Civil War; slavery abortion $57,98,102-3,111-13$, 222

see also reproductive rights academia see intellectuals; universities

Adorno, Theodor 6, 161

Africa 171

African Americans 10, 18, 20, 26-27, 29-34, 71, 94n.32, 99-101, 104-5, 106, 135, 144, 155, 170, 198, 202, 274, 277

"general strike" of, in Civil War 17, 22, 31, 147-48, 151-52, $155,164,203$

women 99-101, 105, 197, 219, $227-28$

see also Black Lives Matter;

Black Panther Party; Black

Power; Civil War; Du Bois; slavery

agriculture 48, 122, 203

see also farmers; land; sharecropping

Alexander, Barbara 53

Algeria 279

alienation 139, 200, 204, 224-25, 228-30

Allen, James S. 33

Alpert, Jane 186-88

Amalgamated Clothing Workers 75
Amalgamated Textile Workers of America 74

“American," meaning of 6

American Civil Liberties Union (ACLU) 74

American exceptionalism xii-xiii, 4, 6-7, 13, 126, 132-34, 264-90

American Federation of Labor (AFL) 26, 74-76, 78-80

American Federation of Teachers (AFT) 74

American Indian Movement (AIM) 210

American Revolution 19, 73, 78, $81,85,122,125$

American Studies 8, 125-26, 136, 140

American Workers Party (AWP) 79-82, 87

American Writers Congress 120

Americanism 5-6, 13, 27, 71-96, $102-4,114,124-25$

and communism 95-115

Americanization of Marxism 20, $61,72,124-25,200$

anarchism 25, 27, 44, 46, 48, 130

anthropology 61

anti-communism 87, 94n.32, 102, 198, 250

see also McCarthyism; Red Scare

Antifada 258

Appeal to Reason 26

Aptheker, Bettina 114 
Aptheker, Herbert 33-34, 38, 202

Arab Spring (2011) 279

Arendt, Hannah 161, 201

art 48, 77, 100, 174

Asia xii, 208, 265, 279

austerity 274-75, 277-80, 282, 284

Austin, J. L. 247

Austria 272, 280

authoritarianism 14, 138, 185

automation 136-37

Ayers, Bill 172-73, 184, 187, 189, 190n.5, 191n.10

Baby Boomers 285

Baffler 248

Baran, Paul 201

Barber, David 173-74

Barrett, Joseph 55

Batkis, Grigorii 106

Baxandall, Rosalyn 103

Beard, Charles and Mary 29, 33-34, 136

Bebel, August 57, 60, 96, 221

Bell, Daniel 14

Bellamy, Edward 204

Benjamin, Walter 252

Berlin, Ira 204

Biederman, Felix 254

Bình, Nguyễn Thị 185

birth control 51, 57, 222

see also abortion; reproductive rights

Black Lives Matter 2, 280

Black Panther Party 170, 176-77, 184

black people see African Americans

Black Power 174, 181

Black Socialists in America (BSA)

$$
\text { 250, } 253
$$

Blackburn, Robin 19

Blair, Tony 247, 274

Blanc, Eric 245

Blight, David 36

Block, Diana 182, 186-87

bohemianism 46, 49-50, 55

Bolivia 278

Bolshevism 58, 76, 98, 102, 106-7, 127-29, 150, 269 see also Lenin; Russian

Revolution; Soviet Union

Bolter, Jay David 241

Boorstin, Daniel 126

Boudin, Kathy 178-79, 187, 189

Boudin, Louis 78

bourgeoisie 20, 29, 33-34, 53, 127, 138, 150, 159-61, 163, 176, 204, 277

Brady, Robert A. 162

Braudy, Susan 189

Brexit 277

Brick, Howard 200

Briggs, Cyril 26, 105

Britain 34, 130, 159-60, 198, 205, 247, 271-73, 275, 277, 282 see also Blair; Corbyn; English Civil War; Labour Party

Bromley, Dorothy Dunbar 113

Brooks, Michael 256

Brookwood Labor College 74-75, 78,86

Brophy, John 78

Browder, Earl 27-28, 95, 97, 104-6, 115n.1, 124

Browder, Raissa 95

Brown, Elsa Barkley 156

Brown, John 21, 25-26, 28

Brown, Kathleen 52

Brownmiller, Susan 220-22, 235

Bruenig, Elizabeth 259

Budenz, Louis 71-94

Buhle, Paul 20, 249, 259

Bukharin, Nikolai 4

Bunche, Ralph 152

bureaucracy 128, 269

Burke, Kenneth 120-21, 124, 129

Burnham, James 80, 120, 123, 126-29, 135

Burrough, Bryan 173

Calverton, V. F. 9, 43, 49-51, 56, $58,60-62,74,77-78,120$

Camus, Albert 199-200, 202

Cannon, James P. 80, 82-83

capitalism xiii, 1-2, 5-6, 10-14, 18, 19, 24, 27, 29-31, 33, 35, 37-38, 41, 46, 49, 55, 60-61, 
73, 77, 79, 80, 83, 96, 103, 105, 108, 113, 119, 121-23, 127-30, 133-38, 140, 147 , 150, 157-60, 163, 170, 174, 176, 189, 195-96, 199-200, 203, 206, 217, 224, 251, 253, 257, 264-68, 270-74, 276, 278-79, 282-83, 285

crises of $267,273,278,282-83$, 286

see also financial crisis; Great

Depression; Great Recession perceptions of 1

varieties of xii

capitalists 10, 25, 29-30, 32, 46, 76, 121-23, 162, 202

Castro, Fidel 177, 185

Catholicism 73-75, 85, 87, 98, 105-6, 185

censorship 26, 124, 235 see also repression

Chapo Trap House 242, 254-59

children 10, 55, 57, 59, 98, 100,

102, 104, 108, 111-13, 124,

172, 202, 205, 220

see also education

Chile 279

China 48, 184, 279 see also Mao Zedong; Maoism

Christianity 26, 86, 280 see also Catholicism; religion

Christman, Matt 254

citizenship 75, 104, 148, 154-55, 234

civil liberties 27, 74, 230

Civil Rights Act (1964) 222

civil rights movement 31,172 , 179-80, 197, 274

Civil War 2-3, 8, 10, 17-42, 73, $78,137,139,148,151-52$, $154,164,203$

civilization $6,29,46,62,103-4$, 106, 136-37, 140, 265

Claflin, Tennessee 45

Clark, Judith 179

Clark, Peter H. 250

class 2, 8, 10-11, 13, 19, 21, 37, $114,133,151,163,173-77$,
180-83, 188, 198, 205-6, 217, 219-24, 234, 236, 260, 274, 282

collaboration 123

conflict 17, 22, 29, 127, 151, 157-58, 202, 210, 265-66, 278 consciousness xiii, 18, 20, 37, $52,54,76,84,120-21,133$, $152,162,175,211,257,264$, 266, 270, 277, 285

employing 100, 274

enemies 102, 107

formation 238n.22, 282

gender as 11

hatred 133

politics 13, 249, 267, 270-71

reconciliation 27

reductionism 10, 146

ruling 32, 148, 159, 204

solidarity $18,22,54,76,81$

struggle $2,4,7,10,25,33,49$, $84,123,250-51,265-66,268$

upper 105,278

war 26, 78

see also classlessness; middle class; proletariat; working class

Class Struggle 48

classlessness 7, 249, 251-52

climate extinction 14, 251, 267 see also environment

Clinton, Bill 274-75

Clinton, Hillary 247, 256

Clover, Joshua 251

Cold War 7-8, 13, 122, 126, 12930, 132-37, 244, 272, 281

liberalism 126, 133-37, 140, 244, 272

Collins, Patricia Hill 227

colonialism 136, 171, 176, 277 see also imperialism

colorblindness 26

Combahee River Collective 249

Comintern see Communist International

Commager, Henry Steele 126

Committee for Non-Violent Action 208 
commodification 30, 180, 219, 227-30, 232, 235

commodities 11, 29, 181, 224-30, $238 n .22$

\section{Commune 251}

communications see media communism 10, 127, 175-76, 180, 198, 251, 266 see also socialism

Communist International 8, 27, $102,114,124,150,270$

Communist League of America (CLA) 80

Communist parties, global 176, 270, 274 see also Communist

International

Communist Party of the United States of America (CPUSA) 7, 9, 11, 27, 48-50, 60, 71, 73, $76,81,83,86-87,95-118$, $120,124-25,128-29,135$, 144, 146-47, 196, 198, 202 and African Americans 26-28 see also Browder; Foster;

Lovestone

Confederate States of America see Civil War

Conference for Progressive Labor Action (CPLA) 74, 76-80, 85,88

confiscation 26, 31

see also expropriation; land, redistribution; redistribution Congress of Industrial Organizations (CIO) 71-72, $74,80,82,86,88$

consciousness xii, 19, 36-37, $49,61,76,120,127,144$, 151-52, 196, 207, 236, 257, $269,277,285$

false 131, 217, 224, 230, 234-35 raising 217, 221, 226, 234 see also class; culture; ideology; philosophy; racism conscription 30 conservatism 2-3, 7, 26-27, 96, 130, 132, 196, 231, 235-36, $249,257-58,274,276-77$ in Communist Party 102, 106-7, 114-15

in labor movement 74-75 see also McCarthyism; Reagan;

Thatcher; Trump

Conservative Party (U.K.) 277

Constitution of the United States 5 , $83-85,202$

consumerism 5, 37, 49, 137, 179-81

consumption 230-34, 242

Corbyn, Jeremy 247, 251, 275, 280

Corey, Lewis 120, 159-60

see also Fraina

coronavirus xiii

Coughlin, Charles 84

counterculture 171, 179, 184

Cowl, Margaret 108

Cowley, Malcolm 49

Cox, Oliver Cromwell 10, 144-69

crime 154, 248

see also prisons

Crosland, C. A. R. 247, 272

Crossman, Richard 198

Cruse, Harold 135

Cuba 171, 175-77, 185

culture xiii, 4, 6-8, 11-13, 20, $36-37,46,49,52-53,58-59$,
$71,74,77,79,84-85,87$,
$125-26,134,136-37,145$,
$196,198,206,218,220$,
$222-23,241-65,276$
see also counterculture

Current Affairs 241, 245, 248, 250

Daily Worker 47-48, 109, 112-13

Danielson, Leilah 200

Davis, Angela 7, 183

Davis, Mike 272

Day, Meagan 259

De Leon, Daniel 25

Dean, Jodi 257

Debray, Regis 177

Debs, Eugene Victor 4, 5, 26, 204

debt 244, 285

deindustrialization 246, 274, 277, 283

Dell, Floyd 43-44, 47-49

democracy $14,18,30-32,38$, $122,126,129,137,146-49$, 
152-54, 156-64, 202,

273, 279

socialist 269

workers 81

see also social democracy

Democratic Party 28, 88, 121, 204, 254, 265, 274-77, 281

see also New Deal

Democratic Socialists of America

(DSA) 1, 244-45, 248, 253, 281

Denning, Michael 8, 71-72, 74, 87, 125,198

deportation 26, 135, 140

depression see capitalism; Great

Depression

determinism 7, 34, 130-31

Deutsch, Helen 222

Devlin, Bernadette 185

Dewey, John 6, 75, 199-200

Di Prima, Diane 252

dialectical materialism 114, 127 , 129-31, 134, 176

see also historical materialism; philosophy

dictatorship 154-55, 160, 177, 285

see also fascism; Nazism

dictatorship of the proletariat 148 , $150-53,176$

Dissent 248

divorce 96, 102

Dohrn, Bernardine 178-87, 189

Dos Passos, John 129

Douglass, Frederick 28

drama 77

Du Bois, W. E. B. 10, 22, 31-33, 38, 120, 135, 144-69, 203

Dunayevska, Raya 17, 138-39

Dunbar-Ortiz, Roxanne 183, 210

Dutt, R. Palme 160

Dworkin, Andrea 11, 218-19, 224-26, 229, 234-35

Dylan, Bob 170

Eastern Europe 124, 128, 269, 277

Eastman, Max 129

Echols, Alice 218

Economist 243-44, 248

Edelman, Elaine 207 education $1,75,77,79,120,148$,

153, 196, 204-5, 207, 212,

245-46, 277, 282

see also children; teachers;

universities

Ehrenreich, Barbara and John

205-6, 245

elections 1, 4, 34, 244, 274, 276-78, 280

Elkins, Stanley 203

Emancipation Proclamation 26

Emerson, Ralph Waldo 126, 131, 200

emotions 45-46, 58-59, 84, 211

empire 8, 12, 27, 34, 37, 86, $175-76,265$

see also imperialism

employment 14, 54, 100, 103-4, 119, 181, 220, 224, 227, 230, 244, 251, 259, 264, 277, 282-83, 285

enclosure 238n.22

"end of history" 248

"end of socialism" (1989) 7

Engels, Friedrich xii, 2-4, 7, 9-10, 19-20, 23, 28-29, 36, 38, 44, 48, 57, 60, 96, 107, 130, 183, 201, 217, 220-21, 265, 267, 278

Communist Manifesto xii, 11, 36, 45, 96, 197, 201, 204, 252, 265-66

German Ideology 10, 204

Origin of the Family, Private

Property, and the State 96, 220-21

English Civil War 29

environment $1,88,248-49$

see also climate extinction; Greens

Epstein, Abraham 78

eroticism 228-31

see also sexuality

ethnicity 10, 18, 20, 22, 62, 71, 73, 246, 277

Europe xii-xiii, 2-6, 11-13, 18, 20, $29,78,83-85,99,107,126$, 132-33, 136, 145, 198-200, 264, 269-80, 282

"Americanization" of 270

see also Eastern Europe 
European Union 275, 277

Evans, Janet 103

Evans, Sara 218

exceptionalism see American exceptionalism

existentialism 13, 199-202, 211 exploitation xiii, 27, 34-35, 52, 54-55, 157, 177, 181, 183, 210, 223-25, 227, 230, 235, 283,285

of women 184, 224, 227, 230, 235

expropriation 3, 206, 273

see also confiscation; redistribution

Facebook 236 see also social media family 9-10, 44, 49-52, 57, 59, $95-115,185,220,222,234$ see also marriage; monogamy;

Engels, Origin of the Family

Fanon, Frantz 176, 217

farmers 24, 28-29, 119

see also agriculture; land; sharecropping

fascism 5, 13, 84-85, 87, 95, 99 , 109, 111, 114, 122, 124, 147, 154, 158, 160-64, 198, 245, 255,277

Faue, Elizabeth 52

Faulkner, William 210

Federal Bureau of Investigation (FBI) 171, 173, 176, 178-79

Fellowship of Reconciliation 86 feminism 2, 11-12, 44, 57, 62, 113-14, 171-74, 178-83, 184-89, 217-40, 279

bourgeois $57,181-82,188$ socialist 172,186 see also gender; women feudalism 3, 29-31, 33, 84, 132-33, 137, 157, 159, 265 see also serfdom

fiction see literature

film xii, 53

see also media

financial crisis (2008) xiii, 1 , 243,278 financialization 13, 35, 246

Finland 128

Firestone, Shulamith 217, 220-21

First International see International Workingmen's Association

First World War 26, 46, 49, 73, 77, 267, 269-70, 278, 282

Fisher, Mark 257

Fitzgerald, F. Scott 130

Flynn, Elizabeth Gurley 87, 111

Foner, Eric 32, 204, 207

Foner, Philip 144, 164, 202

force see violence

Ford, Henry 5

Fordism 73

Foster, William Z. 27

Fourier, Charles 44

Fourth International 86

Fraina, Louis 78, 160 see also Corey, Lewis

France 103, 129, 272, 274-75, 277, 279-80, 282

see also French Revolution; Paris Commune

Frankfurt School 6, 61 see also Adorno; Horkheimer; Marcuse

Fraser, Nancy 258

"free labor" see labor

free love 44-46, 98, 102, 171, 179, 184

freedom 5, 13, 22, 26, 30, 35, 38, $43,47-49,56,59,75,78,83$, 104, 130-32, 134-39, 154, 159-60, 184, 196, 198, 199

Freedom Summer 227

French Revolution 29, 37, 148, 159

Freud, Sigmund 9, 43-44, 46-47, 49-50, 60-62, 217, 220-21, 234

Freudianism 219, 222, 230

Frost, Amber A'Lee 255

Gardener, Tracey 228

Garlin, Sender 48, 112

gay see homosexuality; sexuality gender xiii, 8-12, 22, 48, 55, 59, $96,109,111,173,180-81$, 
184, 189, 217-19, 221,

232, 255

see also feminism; masculinity;

women

Generation X 252, 285

Generation Z 285

Genet, Jean 220

Genovese, Eugene 29

Gerhard, Jane 218

German Americans 7, 9, 18, 22, 45-46

see also Lore; Sorge;

Weydemeyer

German Social Democratic Party

(SPD) 149, 247, 267, 271

Germany 2, 4, 23, 107, 122, 131, 149, 176, 247, 267, 269, 271-73, 275, 280, 282

1848 revolution $11,22,148$ see also Nazism

Ghandianism 86

Gilded Age 4, 23, 34-36, 204, 206

Gitlin, Todd 173, 191n.13

Godwin, William 44

Gold, Michael 43, 60, 120

Goldman, Emma 183, 204

Gooding-Williams, Robert 149

Gordon, Eugene 105

Gorman, William 167n.19

Gosse, Van 98, 103

Gould, Jay 35

Gramsci, Antonio 5-6, 71, 79, 230-31, 256, 267, 286

Great Depression 11, 13, 71-94, 119, 121-22, 127, 150, 264

Great Recession 1, 243-44, 268, 275, 278

see also financial crisis; recessions

Great Society 275

Greece 275, 279

Green New Deal 281

Greenback-Labor Party 24

Greens 280

Gropper, William 100-1

Group of Seven (G7) 268

Gruson, Richard 241

Guérin, Daniel 162

guerrilla war 177, 252
Guevara, Che 177, 252

Gutman, Herbert 37

Hahn, Steven 156

Haider, Asad 249

Haitian Revolution 33, 135

Halbwachs, Maurice 37

Halper, Katie 256

Hardman, J. B. S. 75-76, 78, 80-82

Harrington, Michael 251

Harris, Abram 151

Harris, Malcolm 245

Harrison, Hubert 26, 147

Hartmann, Heidi 182, 188

Hartz, Louis 15n.8, 132-33, 248

Hawthorne, Nathaniel 126

Hay, Harry 106

Hayden, Casey 180, 182

Hayek, Friedrich 158, 160-61, 163

Haymarket 2, 25, 204

Haywood, Bill 99

healthcare $1,5,112,205,251$, 281,284

hedge funds 13

Hedges, M. S. 77

Hegel, G. W. F. 127, 131

Henry, Patrick 85

Herberg, Will 150-51

Herndon, Angelo 28

heterodoxy 8-9, 147, 161

heteronormativity 222

higher education see universities

Hill, Christopher 29

hip-hop 252-53

historical materialism $6,25,47,49$, 61-62, 97, 132, 195-96, 199, 206, 212, 220, 252

see also determinism; dialectical materialism; Marxism

historiography 19, 29, 144, 147, $151,155,157,195-216$

history xii, $35,77,85,131$, 195-216, 265-67, 270

Hitler, Adolf 132, 161, 198

Ho Chi Minh 185

Hobsbawm, Eric 34, 37, 123

Hofstadter, Richard 132

Hollibaugh, Amber 226-27, 235 
Holocaust 161 see also Nazism

Holt, Thomas C. 203

homelessness 14, 119

see also housing

homosexuality $62,96,102,106-7$, 222, 227, 231 see also sexuality

Hong Kong 279

Hook, Sidney 6, 74, 78, 80-81, $120,124,127,135,200$

hooks, bell 219

Horkheimer, Max 6

Horney, Karen 222

hours see workday

House Committee on Un-American Activities (HUAC) 85, 87

House of Representatives 1, 244

housing 14, 244, 248, 277

Hudson, Hosea 28

humanism 13, 121, 131, 138, 200

Hungary 138

hunger 60, 119

Hutchins, Grace 100

identity xiii, 20, 34, 37-38, 72, 85, 211-12, 249, 255

see also class; gender; nationalism; race

ideology 6, 8, 22, 30, 32, 35, $46,50,67,77,85,96,105$, 120-21, 132, 149, 156, 162, 176, 187-88, 198, 201, 209, $225,232,242,250,255$ see also consciousness; Marx,

German Ideology

immigration 2, 6-9, 20, 73, 124-25, 133, 138-40, 196,

198, 246, 248, 277, 280 see also deportation; migration imperialism 31, 75, 84, 128-29, 131, 151, 157, 170, 173, 176-77, 179, 182-83, 187-89 see also colonialism; empire In These Times 248

income 32, 108, 153, 282, 284-85

Indians see Native Americans
Industrial Workers of the World (IWW) 26, 123

industry see capitalism; manufacturing

inequality $1,13,19,23,59,62,88$, $95,108,144,146,158,163$, 195-95, 198, 205, 223, 234, $264,273,275,279,282$

inflation 108, 273

insurance $14,75,78$

intellectuals 7, 32, 35-36, 71, 74, 78-80, 83, 87, 119-69, 206, 217, 231, 234-35

see also New York Intellectuals International Brotherhood of Electrical Workers 77 International Women's Day 101 International Workingmen's Association 3, 8, 18, 23, 27, 45 internationalism $8,19-22,31,38$,

Iraq 279 $72,75,80,83,85,87,195$

Irish Americans 45

Isserman, Maurice 124

Italy 5, 122, 269, 272, 274, 277, $279-80$ see also Gramsci

Jacobin 2, 241, 244-45, 247-48, 251,255

Jacobs, John (J.J.) 175

Jaffe, Naomi 178-81

James, C. L. R. 12, 33, 120, 135-40, 146, 156, 167n.19

James, Joy 149

James, William 199

Janney, Caroline 36

Japan 271

Jefferson, Thomas 78

Jews 12, 196

Johnson, Boris 277

Johnson, Walter 29

Johnstone, Jenny Elizabeth 108

Jones, Claudia 120, 147

Jones, Jeff 187

Jospin, Lionel 274

journalism 77, 83, 246

see also media 
Kazin, Michael 32, 208

Kelly, Brian 151-52

Kent State 197

Keynesianism 274

King, Mary 180, 182

Klein, Bonnie Sheer 229

Klonsky, Mike 174-76

Knights of Labor 24, 25

Koestler, Arthur 198

Kollontai, Alexandra 4, 51-52, 59-60, 96

Kornhauser, William 161

labor xiii, 3, 17-22, 25-32, 34-36, $38,45-48,60,71,74,77-78$, $81,87,98,122-23,139,151$, 153-55, 203, 224, 226, 246, $248,265-66,268,283,285$

division of 206, 220-21, 223

"reserve army of" 264

see also unions; wage labor; work; working class

Labor Action 74, 76, 81, 87

Labor Age 74-75, 77

labor movement xiii, 7, 13, 21-22, $72-73,75,77,80,86,100$, 123-24, 150, 265, 271 see also unions; working class labor party 4, 19, 73, 75, 265, 276 Labor Reform Association 22 labor unions see unions

Labour Party (U.K.) 271-72, 275, 280

land 19-20,33,119, 148

ownership 24, 133

redistribution $26,28,31-32,154$

Laos 175

Latinos 274

law 5, 21, 32, 35, 57, 98, 102, 106-7, 111-13, 159, 178, 204-5, 222-23, 232-34, 236, $238 n .22$

Lebanon 279

Lebedeva, Vera 112

Lefebvre, Georges 29

Left Opposition 129 see also Trotskyism

Lenin, V. I. 28, 129, 267
Leninism 82-83, 85-86, 153, 170, $174,176,199$

Lewis, John L. 79

LGBTQ+ see homosexuality; sexuality

liberalism 2, 6, 11, 12, 22, 34, 73, 124, 126, 132-37, 140, 144, 243, 246-48, 250, 254-55, 258-59, 273-74, 285

see also Cold War; neoliberalism; New Deal; reformism; social democracy

Lincoln, Abraham 17, 18, 21 , $25-28,34,125$

literature $43,52,56,77,120-21$, $130,145,147,150-51,228$, 241, 252 see also poetry

Locke, John 132

London, Jack 198

Long, Huey P. 84

Lorde, Audre 219, 228-29, 235

Lore, Ludwig 78, 80

Lovestone, Jay 7, 133, 150

Luxemburg, Rosa xiii, 12, 160, $245,267,282$

lynching 26-27, 101

Lynn, Denise 103

McAlevey, Jane 259

McCarthyism 3, 124 see also anti-communism; Red Scare

McCullough, Celeste 179

MacKinnon, Catharine 10, 218-19, 222-23, 230, 234-35

McNally, David 279

Mailer, Norman 220, 231

Manson, Charles 172, 179

manufacturing 29,119, 160, 282-84

Mao Zedong 177, 217, 252

Maoism 170, 177, 184

Maranto, Gina 210

Marcuse, Herbert 7, 17, 61, 138, 170, 199, 201, 217

marriage 44-45, 47, 54-55, 57, 59, $96,98,102,105,183$

see also family 
Martin, Ron 229

Marx, Jenny xii

Marx, Karl xii, 2-4, 7, 17-23, 26, $28,29-30,34,36,38,43$, $45,47-50,57,60-62,76$, $96,103,119-43,176,183$, 199-200, 204-5, 209, 217, 235, 243, 249-53, 264-68, $269,277-78,282-83$

Capital 2, 3, 13, 17, 18, 130, 134, 138-39, 199, 201, 225, 265-67, 282

Communist Manifesto xii, 11, 36, 45, 96, 197, 201, 204, $252,265-66$

Economic and Philosophical Manuscripts 138, 201

Eighteenth Brumaire of Louis Bonaparte 19, 134, 201, 266 German Ideology 10, 204 Grundrisse 201, 266

"Theses on Feuerbach" 199

Marxism, definition of 6-7

Marxism-Leninism 170, 174, 176 see also Leninism; Maoism masculinity 55, 67n.60, 226, 233 Mathiessen, F. O. 126

Maurer, James 78

means of production see production

Means TV 258

media 227, 232, 236, 241-63 see also film; journalism

Melville, Herman 126

Melville, Sam 186

memory 19-21, 24, 35-36, 38

Menaker, Will 254, 258

Menand, Louis 13, 131

MeToo movement 2, 218, 235-36 see also sexual harassment

Mickenberg, Julia L. 98

middle class 3, 27, 29, 36, 46-47, 73, 88n.1, 100, 107, 113, 172, 188, 191n.10, 205-6, 244, 272, 275, 277, 279, 281, 283-85

see also bourgeoisie;

professional-managerial class
Middle East 279

migration 133

see also immigration

Milburn, Keir 243

Miliband, Ralph 205

militarism 21, 75

millennials 11, 241-63, 281, 284-85

Miller, Henry 220

Miller, Loren 152

Miller, M. H. 244

Millett, Kate 115, 217, 220-21, 235

Mills, C. Wright 170

misogyny 217, 225, 231, 236

Mitchell, Louise 113

Mitterrand, François 274

Modern Monthly 82

Modern Quarterly 47, 78

modernism 77, 96, 198

modes of production see production

monarchy 150,154

monogamy 47, 49, 51, 171, 179, 183-85

see also family

Montgomery, David 32, 37

Monthly Review 163, 208, 248

Moon, Henry Lee 152

Morais, Herbert 29

Moral Majority 218, 236

morality 43-47, 49, 51, 58, 60, 77, $96,105,107$

Morgan, Robin 179, 188

Moscow Trials 128

motherhood see family

Moylan, Mary 185

Mullen, Bill 148

Murray, Pauli 146

music $6,77,241,252$

Muste, A. J. 13, 71-94, 200

Myrdal, Gunnar 163

n+1 241, 244-45, 248

National Association for the

Advancement of Colored

People (NAACP) 146, 150, 156

National Labor Union 18, 24 
National Organization for Women (NOW) 183

nationalism 21-22, 26, 37, 62, 72, $87,102,126,156,249$

nationality 10

Native Americans 30, 210

Nazism 6, 122, 131, 161 see also fascism; Hitler

Nazi-Soviet Pact 110, 124, 128, 198

Nearing, Scott 43, 48

Nelson, Bruce 158

neoliberalism xiii, 35-36, 158, 235, 242-43, 248-49, 257, 270, 274-75, 278-79, 282, 284-85

Netherlands 280

New Deal 11, 71, 88, 121-27, 129, $132,134,140,275$

New Left 7, 12, 62, 144, 170-94, 195, 198-203, 208, 211, 217, 220, 226, 234, 249, 256

New Left Notes 181, 183

New Masses 27, 47, 54-55, 152

New Politics 245, 248

New Right 7, 219, 235

New York Intellectuals 71

New York Radical Feminists 220-21

New York Radical Women 220

New York Times 244, 259

New York Tribune 2, 17

Nichter, Matthew 150

Niebuhr, Reinhold 120

Nkrumah, Kwame 252

nonviolence see pacifism

Norse, Harold 106

nuclear weapons 267

Obama, Barack 243, 274

Ocasio-Cortez, Alexandria 1, 244, 280

Occupy Wall Street 1, 244, 248, 279-80

October Revolution see Russian Revolution

Old Left 7, 62, 83, 87, 195, 200-3, 211,249

see also Communist Party;

Trotskyism opportunism 12, 73

orthodoxy 8-9, 22, 36, 127, 160, 250

Owen, Robert 44

pacifism $86,94 \mathrm{n} .32$

Paglia, Camille 236

Palme, Olaf 251

Palmer, Bryan D. 114

Pan-Africanism 33

Panken, Wendy 179

Paris Commune 37, 265

Park, Robert 162-63

parliamentarianism $4,73,76,83$, 267, 273

Partisan Review 128

partisanship 22

patriarchy $37,49,51,60,96$, $98,105,178,182-83,189$, $220-22,224,229$

patriotism 22, 26, 84-85, 96, 104, $125,131,204$

see also Americanism; nationalism

peasants 37,154

Peck, Jamie 258

Peck, Raoul xii

Pelosi, Nancy 256

pensions 14

People's Party 24

Pepper, William F. 220

permanent revolution see revolution

Pesotta, Rose 86

Phillips, Ulrich B. 203

Phillips, Wendell 32

philosophy 127, 151, 159, 201, 265

see also consciousness; dialectical materialism; existentialism; morality; pragmatism

Pittman, John 106

Piven, Frances Fox 211

poetry $77,121,229,241,251-52$, 257

Popular Front 9, 11, 52, 59, 71-72, $74,82-83,87,95-115$, 124-26, 129 
population 104, 133, 204, 279 , 283

populism 13, 24-25, 46, 71, 84, $161,251,277,285$

pornography $218-19,222-36$

Portugal 275, 280

Poulantzas, Nicos 205

poverty $52,55,74,104,154,175$, 188,285

pragmatism $6,21,74-77,81,83$, $146,199-200$

praxis $14,76,184,220,250$

prisons $5,199,205,258$

see also crime

production $5,25,35,44,223-25$, $229,232,238$ n.22, 242, 283

means of 2, 7-8, 10, 130, 208, $250,269,273$

modes of 19, 50, 97, 131, 253, 265

productivity $10,271-72,275-76$

professional-managerial class

205-6, 245, 248, 255

see also class; middle class

profit 10, 19, 30, 150, 176, 200,

203, 225-26, 235, 252,

266-68, 271, 282, 284

Progressive Era 35-36, 149, 204

Progressive Labor (PL) 170, 176-77

proletariat 10, 19, 33, 37, 47,

$127-28,134,138,148,152$,

$159,249,252,277$

see also working class

property $10,26,43,45,47,51$, 58-60, 96, 151, 154, 160,

210, 226, 264, 273

prostitution 222, 224, 227

psychology 43-44, 49-50, 61-62, 84

see also Freud

Puerto Rico 279

puritanism 46, 48-49, 67n.60

Quinby, Bryan 255

race xiii, $3,8,12,18,22,26$, $27,57,114,135,144-47$,
$149,157-58,163,173-74$, 180-83, 189, 195-96, 198, 202-3, 212, 219, 248, 274, 284-85

see also African Americans; segregation; whiteness racism $24,26-27,138,174,177$, 182, 187, 198, 203, 212, 277 see also segregation; whiteness radicalism 9, 12, 20, 22, 24-26, 35-36, 44-45, 47, 49-50, 60-61, 84, 96, 98, 100, 104, $119,156,195-96,220,223$, $235,251,256,280$

radio see media

Ramparts 180-81

rape $218,220-23,231,235$

Reagan, Ronald 13, 218, 274-75

recessions $267,274,282$ see also Great Recession

Reconstruction 18-20, 24, 28, $30-32,35,38,147-57,164$, 203

Red Army Faction 176

Red Scare (1919) 2, 26, 75, 77, 96 see also anti-communism; McCarthyism

redistribution of wealth $1,30,32$ see also confiscation; expropriation; land, redistribution

Redstockings 220, 227

Reed, Adolph, Jr. 149, 156, 256 reformism 11-12, 14, 28, 86, 107, $231,247,251-52,273-74$ see also liberalism; social democracy

Reich, Wilhelm 61 reification 181, 224, 234 religion xiii, 10, 21, 44, 52, 57, 73, 84, 86-87, 96, 98, 105, 205 see also Catholicism; Christianity religiosity 9 repression political 26, 106, 124, 131, 138-40, 187, 211 see also McCarthyism; Red Scare 
sexual 48, 61, 217, 220, 229-30

reproduction $44,220,222$

reproductive rights 227

see also abortion

Republican Party 22, 26, 30, 204, 254, 281

republicanism $22,24,161$

revolution 3-4, 11-14, 19, 22, 24, $26,29,46,73,77-79,82-83$, $87,120,127,134,245,249$, 252-53, 260, 265, 269

of $184811,22,148,198,202-3$, 212, 265-66

bourgeois $19,28,30,33,159$

permanent 30,127

proletarian 18

socialist 4, 83, 119-20, 129,

133-34, 179, 182-84

see also American Revolution;

French Revolution; Germany;

Haitian Revolution; Russian

Revolution

Revolutionary Youth Movement II

(RYM II) 176-77, 183

Riley, Boots 252

Robinson, Cedric 145-49, 157

Robinson, Lois 233

Robinson, Nathan J. 245, 250

Rocha, Mona 173

Rockwell, Bruce 210

Roediger, David 148

Roiphe, Katie 236

Roman Empire 265

Roosevelt, Eleanor 122

Roosevelt, Franklin Delano 79, 121-24

Rorty, James 78

Rosen, Ruth 218

Rosenberg, Arthur 160

Rossiter, Clinton 134

Rubin, Gayle 219, 224, 226, 231-35

Rudd, Mark 174, 187

Russia 18, 128

see also Soviet Union

Russian Revolution (1917) 4, 37, 47, 50, 56, 96, 127, 129, 269

Ryan, Erica J. 96
Sandberg, Sheryl 236

Sanders, Bernie 1, 244-45, 247-48, 251, 253, 258, 280-81

Saposs, David 78

Sartre, Jean-Paul 201

Schlesinger, Arthur, Jr. 132

Schmalhausen, Samuel D. 9, 50-51, 60-62

Schroeder, Gerhard 274

Scialabba, George 207

Scott-Heron, Gil 241

Scottsboro Boys 99

Second International 4, 27, 267, 270 see also social democracy

Second World War 110-11, 122, 124, 129, 196, 198, 264, 271

sectarianism $11,44,61,74,76$, 79-81, 87, 133

segregation, racial 31, 37, 135, 274 see also race

serfdom 18, 158, 160-61

service sector 283

sex work see prostitution

Sexton, Tom 258-59

sexual exploitation 184

sexual harassment 52, 55, 218, 222-23, 230, 232-35

see also MeToo

sexual objectification 181

sexual revolution $45,50-51,57$, 59-62

see also free love

sexuality xiii, 8-10, 43-70, 95-115, 171, 181, 184-85, 217-40

see also eroticism

Shachtman, Max 80, 83

Shakur, Afeni 185

sharecropping 5, 30, 32

Shaw, George Bernard 48

Silber, Nina 28

Sinclair, Upton 43, 47-48, 198

Singer, Daniel 275

slavery 3, 17-42, 99, 134, 138-40, 147, 153-55, 196, 202-3, 206, 209, 222, 265, 277

see also abolitionism; African

Americans; Civil War 
Small, Sasha 53, 99-100

Smedley, Agnes 55-56

social democracy xiii 1-2, 4, 36, $149,243,247,251,253-54$, 270-77, 280-82, 285 see also German Social

Democratic Party; reformism; socialism

social media 241

see also Facebook; media;

Twitter; YouTube

socialism $2,7,12,24,36,78,95$,

$125,127,138,148,150,174$,

$176,241-63,267,269,281$

inevitability of $265,267-69,278$

"scientific" 46, 250

see also "end of socialism"; utopian socialism

Socialist Labor Party (SLP) 25, 27, 46

Socialist Party of America 4, 7, 12 , $27,46,73,76,78-79,82,86$, $144,149-50$

see also Debs

Socialist Workers Party (SWP) 60

sociology 146, 157-65, 278

Sombart, Werner 13

Sorge, Friedrich 45

Soviet Union 7, 43, 48, 51, 56-59, 75, 80, 84, 95-96, 103, 105, $107,110-11,122,124$, 126-29, 134, 138-39, 146, $150,198,256,269-70$

Spain 13, 275, 279-80

Stalin, Joseph 7, 9, 71, 87, 95, 102, 105-6, 110, 126, 128-29, 132-34, 140, 269-70

Stalinism 28, 86, 88n.1, 128-29, $131,198-99,201,269-70$

Stalinization 111

Stampp, Kenneth 32

Stanton, Elizabeth Cady 28

state $34,57,88,102,123,128$, 177, 204-5, 253, 259, 269, 273,275

welfare 271-72, 275

see also Engels, Origin of the Family
Steinbeck, John 198

Stern, Susan 182, 186

Stevens, Thaddeus 35

Steward, Ira 22

Stolberg, Benjamin 150

Stone, Hannah M. 112

Strachey, John 159-60, 162

Streeck, Wolfgang 275

strikes $2,5,10,74,78-79$, 85-86, 99, 101, 245-46, 251, 273-75, 279-82, 284

Electric Auto-Lite (1934) 72, 80-83

Great Railroad (1877) 24

Lawrence, Massachusetts (1912) 99

Lawrence, Massachusetts (1919) 73-74

San Francisco (1934) 99

sit-down 86

slaves' $17,22,31,147-48$, 151-52, 155, 164, 203

students' 175

teachers' 245,280

women's 99-102, 280

Strong, Anna Louise 58

Student Non-Violent Coordinating Committee (SNCC) 197

Students for a Democratic Society (SDS) 170, 172-78, 180-81, 189

suffrage $98,148,154-55$

Sunkara, Bhaskar 244, 247, 250-53, 259

Supreme Court 222

surplus value 10

Sweden 251, 272, 275

Sweezy, Paul 160, 163, 201

Sylvis, William H. 24

Tanner, Caroline 179

taxation 11, 284

Taylor, Frederick Winslow 5

Taylor, Keeanga-Yamahtta 156

teachers 205, 245-46, 279-80, 283-84

see also American Federation of

Teachers; education 
technology 51, 199, 241-43, 252, 257, 259-60, 265, 267, 273, 275,282

see also production, means of

Teish, Luisah 227-28, 235

teleology 19, 127, 150, 265

television see media

Thatcher, Margaret 274-75

Third International see Communist International

Third Period 59, 76

Third World 170-71, 176-77, 187

Thompson, E. P. xii, 37, 266

Thoreau, Henry David 126

time see workday

Tlaib, Rashida 244

Tocqueville, Alexis de 132

totalitarianism 6, 132, 134, 137, $198,200,270$

trade unions see unions

tradition 37

transcendentalism 20, 126

Traverso, Enzo 19

Trillbilly Workers Party 256-58

Trotsky, Leon 4, 6, 37, 87-88, 127-29, 160, 170, 270

Trotskyism 60, 80, 85, 92n.17, 128-29, 199

Trump, Donald J. 13, 244, 254-56, 277

Truth, Sojourner 28, 99

Tubman, Harriet 28, 99

Turner, Frederick Jackson 133

Twitter 250, 255

see also social media

\section{Unemployed Citizens' Leagues} 79-81

unemployment see employment unions 4, 14, 24, 36, 46, 72-80, 84-85, 87, 109, 123, 246, 271-72, 274-75, 277, 279-80, 283-84

see also American Federation of Labor; Congress of Industrial Organizations; labor movement; strikes; working class
United Auto Workers (UAW) 81

United Farm Workers (UFW) 227

United Kingdom see Britain

United Mine Workers of America (UMWA) 79

universities 1, 36, 126, 146, 150, 161, 178, 196-97, 201, 210-11, 220, 244, 246

utopian socialism 2, 4

value 10,268

Vance, Carole 217-18

Vance, J. D. 256

Varon, Jeremy 173, 184

Venezuela 278

veterans 20-21, 24, 27, 36

Vietnam 171, 175-77, 189

Vietnam War 86, 174, 181, 185, 197

Village Voice 231

Vinson, Mechelle 223

violence 20, 26, 31-33, 52-53, 55, 170-77, 180, 204, 218-24, 227-28, 231-32, 234-36, 259

see also lynching; repression

vote see suffrage

Wade, Benjamin F. 18

wage labor 24, 34, 208, 210, 268

wage slavery $20,24,30,35$

wages $10,45,100,104,134,137$, $155,266,268,271,276-77$

Walker, Alice 228

Wallace, Mike 208

Wallerstein, Immanuel 157

Warren, Elizabeth 245

Washington Post 259

Weather Underground 170-75, 182-83, 186-88

name variations $190 \mathrm{n} .3$

Weatherman 11-12, 177-88

Weatherwomen 174, 179-80, 183, 186-89

Weeks, Jeffrey 60

Weimar Republic 161

Weisbord, Albert 48

Weiss, Benjamin 48

Weydemeyer, Joseph 22-23 
white supremacy see race; racism; segregation

whiteness 22, 25-26, 145, 151 see also race; racism

Wicks, H. M. 48

Wilkerson, Cathy 171, 178-79, 183-84, 186, 188

Willis, Ellen 219, 221, 227,

$$
\text { 230-32, 234-35 }
$$

Wilson, Edmund 129, 134

Winant, Gabriel 245

Winston, Henry 104

Winter, Ella 57-58

Wollestonecraft, Mary 44

women $5,10,12,20,45,48,51-$ 59, 95-118, 138-40, 170-93, 203, 217-40, 279-81, 283

African American 99-101, 105, 197, 219, 227-28

see also feminism; gender

Women Today 109-11

Wood, Charles 43, 48

Woodhull, Victoria 45

work 133

workday 14, 138-39, 227

Workers' Party of the United States $82,85,87$

working class xii-xiii, $2-3,10,18$, $20,22,25-27,30,34,36$, 43, 46-48, 71-77, 81, 83-85, 102, 110, 122-23, 130, 137, $139,150-51,155,163-64$, 175-77, 196, 198, 204-6, 245-46, 249, 255, 259, 264, 266-67, 269, 271-78, 281, 283-85 black 148, 150, 198

movement xii, 264

organization 3, 109, 266, 269, 271,285

politics 13,271

power 12

solidarity 81

white $148,177,277-78$

women $55,100,113,279$

see also class; proletariat

Working Women 53-54, 100-102, 109

workplace 14, 30, 34, 52, 137, 218, 223, 233-35, 246, 259, 268

World League for Sexual Reform 107

World War I see First World War

World War II see Second World War

Wright, Frances 44

Wright, Richard 135, 145, 198

Yippies 62

Young Communist League 104

Young Marx (film) xii

Young People's Socialist League 244 youth 1, 14, 57, 103-4, 147, 172, 175-76, 179, 200, 251, 283 see also children; millennials; Revolutionary Youth Movement II

YouTube 256

Zetkin, Clara 96, 107

Ziegler, Phil E. 78

Zinn, Howard 13, 195-216 